\title{
Grasping force estimation for prosthetic hands via feature extraction of surface EMG
}

\author{
Lisha Xu, Gaoke Zhu and Xiaogang Duan ${ }^{\mathrm{a}}$ \\ School of Mechanical \& Electrical Engineering Central South University Changsha, 410083, China
}

\begin{abstract}
A prosthetic hand with a self-regulated grip force could achieve different operation modes, which can help the upper limb amputees to fetch objects of different shapes. To get the appropriate grasping force with smaller samples and shorter training time, the method of threshold value judgment in this paper is effective on achieving the estimate of the discrete force basing on the mean absolute value (MAV) of EMG's level. The 10 subjects can be divided into 8 grasping patterns determined through three levels: the small, medium and great of grasping forces in experiments. Experimental results conditioned on small training samples and short training time show that the accuracy of force estimation is $72.91 \pm 9.58 \%$ and thereby convincing the effectiveness and reality of the proposed method.
\end{abstract}

Keywords: force estimation; feature extraction; surface EMG; prosthetic hands.

\section{Introduction}

The aim of most modern multi-freedom prosthetic hand's electrical control systems is to control the action mode of prosthetic hand, but few commercial SDOF prosthetic hands adapt open-loop proportional control which is based on EMG [1,2] to regulate the grasping force and velocities of clogging and releasing. Upper-limb amputees are not only desired to realize different grasping postures by prosthetic hands but also more in need of classifying the prosthetic hand's grasping strength through the hardness and weight of grasped objects.

EMG signals can be measured on the surface or by needle electrodes that are injected into the subject's arm. The EMG signal recorded at the surface of the limb is known as surface EMG (sEMG),which are from skeletal-muscles are a convenient means for users who do not want to deal with additional surgeries and regular hygiene problems. The sEMG can be used to control the force of the hand prosthesis.

Currently, for methods used to estimate the force, one of them is the method based on the muscle model [3], in which the relationship between the muscle force and the EMG is established and then the joint torque is estimated by the muscle-skeletal model. However, because of the complexity of human physiological structure (i. e. the uncertainty of every individual), it is difficult to establish an accurate model of each individual. There are many other methods based on the statistical regression approach to establish the function relationship between grasping force and EMG signals, such as polynomial regression[4], neural network [5,6], SVM[7], and so on. Unfortunately, a large number of samples are needed, and continuous forces decoding elements are separated from the EMG signal

${ }^{\text {a }}$ Corresponding author: xgduan@csu.edu.cn

(C) 2016. The authors - Published by Atlantis Press 
in these methods. Because the nonstationarity of the EMG will lead to low reliability, such as longstanding collection, the minute change of electrode position and muscle fatigue which will seriously affect the accuracy of prediction.

In this paper, the method of threshold value judgment is proposed to realize the discrete force decoding. We find the obtained grasping forces which based on the level of EMG's mean absolute value (MAV). The experiment classifies 10 subjects into 8 dissimilar grasping patterns by the extents of small, medium and great grasping forces and validates the advantage of smaller training samples and shorter training time. Experimental results show the effectivity of the proposed method.

\section{EMG feature extraction}

Grasping force reflects the level of muscle contraction. Usually, the stronger the grasping force is, the deeper the level of the muscle contraction is and the greater the EMG signal energy is. As a result, the characteristics that reflect the energy level in EMG signal can be extracted to represent the grasping force.

The success of a force estimation system depends almost entirely on the choice of features representing the data sequence. The EMG signal can be represented in various forms or parameters. Different forms or parameters result in different analytical complexity and functional advantages. The algorithms for EMG feature's extraction or parametric representations are described below.

By the literature [8-10], there are 13 kinds of characteristics about EMG signal energy (table1) that can reflect the different grasping force levels. The traditional parametric features of EMG signals include integrated EMG variance, Mean value of absolute value, variance, Root mean square, V order detector, Log detector, Sum of absolute value, Mean power, Zero order spectral moment, First order spectral moment and Two order spectral moment parameters. They are all from the real-world processes. However, computational complexity is the major concern. All of them can be computed in real-time. For each feature $\mathrm{u}(\mathrm{k})$ denotes the kth sampling data in the window and $\mathrm{N}$ is the window length for computing the features.

Integrated EMG (IEMG): This parameter is found by calculating the summation of the absolute values of EMG signals. It can be treated as a signal power estimator. It is defined as

$$
I E M G=\sum_{k=1}^{N}|u(k)|
$$

Mean value of absolute value (MAV): This parameter is used to estimate the power mean value of the absolute value of EMG signals. Its definition is given by

$$
M A V=1 / N \sum_{k=1}^{N}|u(k)|
$$

Modified mean absolute value type 1 (MAV1): it is an extension of MAV feature. The weighted window function wkis assigned into the equations for improving robustness of MAV feature. It is calculated by

$$
M A V 1=1 / N \sum_{k=1}^{N} w(k)|u(k)|
$$

Where

$$
w(k)=\left\{\begin{array}{l}
1, \quad 0.25 N \leq k \leq 0.75 N \\
0.5, \quad \text { otherwise }
\end{array}\right.
$$


Modified mean absolute value type 2 (MAV2): MAV2 is an expansion of MAV feature which is similar to the MAV1. However, the weighted window function wi that is assigned into the equations which are continuous functions. It improves smoothness of the weighted function. The equation is defined as:

$$
M A V 2=1 / N \sum_{k=1}^{N} w(k)|u(k)|
$$

Where

$$
w(k)=\left\{\begin{array}{l}
1, \quad 0.25 N \leq k \leq 0.75 N \\
4 k / N, \quad k<0.25 N \\
4(k-N) / N, \quad k>0.75 N
\end{array}\right.
$$

Variance: This parameter is used to estimate the power density of the EMG signal. Its definition is given by

$$
V A R=1 /(N-1) \sum_{k=1}^{N} u(k)^{2}
$$

Root mean square (RMS): It is another popular feature in the analysis of the EMG signal. It is modeled as amplitude modulated Gaussian random process which relates to constant force and non-fatiguing contraction. It is also similar to standard deviation method. The mathematical definition of RMS feature can be expressed as

$$
R M S=\sqrt{1 / N \sum_{k=1}^{N} u(k)^{2}}
$$

$\mathrm{V}$ order detector (v-Order): It is defined from a functional mathematical model of the EMG signal generation. The mathematical definition of the $\mathrm{V}$ feature is defined as

$$
v \text { Order }=\sqrt[v]{1 / N \sum_{k=1}^{N}|u(k)|^{v}}
$$

Log detector $(\log \mathrm{D})$ : This feature also provides an estimate of the muscle contraction force, which can be defined as

$$
\log D=\exp \left(1 / N \sum_{k=1}^{N} \ln |u(k)|\right)
$$

Simple square integral (SSI): It is a summation of square values of the EMG signal amplitude. Generally, this parameter is defined as an energy index, which can be expressed as

$$
S S I=\sum_{k=1}^{N}|u(k)|^{2}
$$

Mean power (MNP): MNP is an average power of the EMG power spectrum. The calculation is defined as

$$
M N P=\int_{0}^{\infty} P(f) d f / M
$$

$\mathrm{P}(\mathrm{f})$ is the EMG power spectrum at frequency bin $\mathrm{f}$, and $\mathrm{M}$ is the length of the frequency bin. 
Zero spectral moment (SM0):SM0 is defined as an aggregate of the EMG power spectrum. The definition is as

$$
S M 0=\int_{0}^{\infty} P(f) d f
$$

The 1st and 2nd, Spectral moments (SM1, SM2): Spectral moment is an alternative statistical analysis way to extract features from the EMG power spectrum. The first three moments (SM1-SM2) are the most important spectral moments. The definition of their equations can be expressed as

$$
\begin{aligned}
& S M 1=\int_{0}^{\infty} P(f) f d f \\
& S M 2=\int_{0}^{\infty} P(f) f^{2} d f
\end{aligned}
$$

\section{Searching optimal representative EMG features}

\subsection{EMG data acquisitions}

In order to extract the best characteristics to analyze the grasping force, the experiment is divided into small force, medium force, and vigorously force with three rounds. Each experiment round includes free state, sphere grabbing, cylinder grab, three fingers grab, two fingers grab, aside grab, hook grab and hand grab 8 kinds of action modes in all. On circulation two and a half, every action holds for 5s. There are 20 actions in each round and finally capturing the EMG signal data for analysis in every movement of the period from $1501 \mathrm{~ms}$ to $4251 \mathrm{~ms}$.

As shown in Figure 1 to Figure 13,after three rounds of grasping experiments containing 13 features, it's the characteristic value of 640 samples of the same subjects (participants 5) in each round of 16 action (8 species). In Figure 1 to Figure 13, every movement contains 40 analysis windows, there are 40 motion samples between the two adjacent vertical dashed lines. The analysis window is $256 \mathrm{~ms}$, the sliding addition is $64 \mathrm{~ms}$ and every movement lasts for $2.75 \mathrm{~s}$; The characteristic value armband has eight electrodes, namely the EMG signal has eight channels, each action sample take the mean of 8 channels characteristic value from Figure 1 to Figure 13.

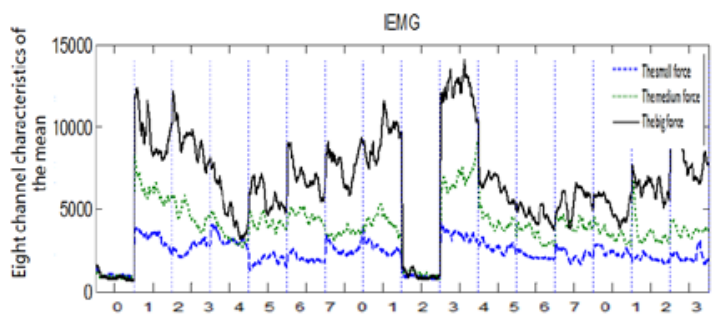

0-Free,1-Sphere, 2-Cylinder,3-Three fingers,4-Two fingers,5-Sides,6-Cusp,7-Phone

Figure 1. The action sample of IEMG amplitude characteristics under different levels of grip force

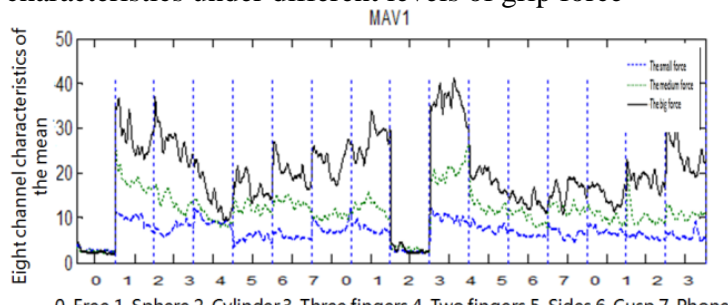
0-Free,1-Sphere,2-Cylinder,3-Three fingers,4-Two fingers,5-Sides,6-Cusp,7-Phone

Figure 3. The action sample of MAV1 amplitude characteristics under different levels of grip

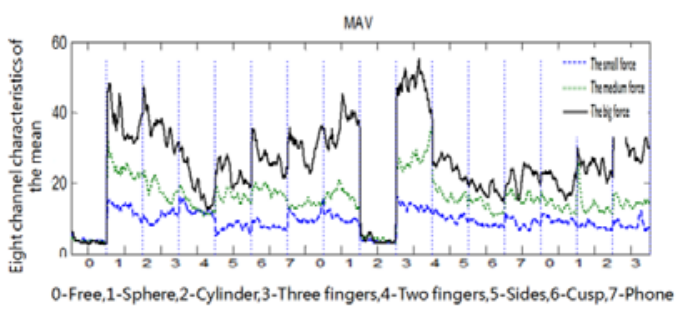

Figure 2. The action sample of MAV amplitude characteristics under different levels of grip force MAV2

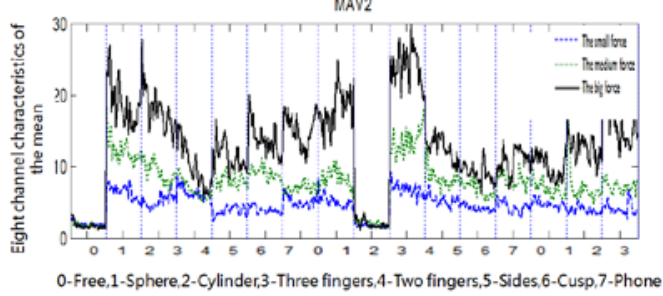

Figure 4. The action sample of MAV2 amplitude characteristics under different levels of grip force 


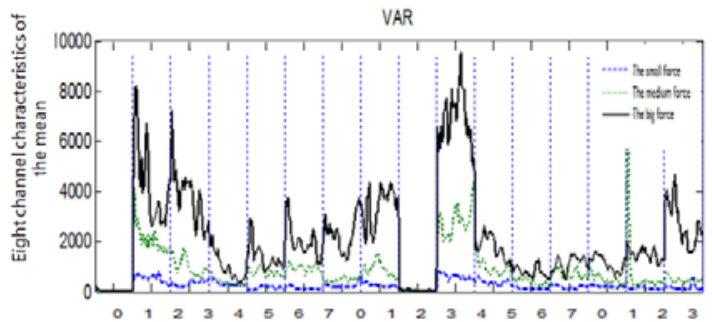

0-Free,1-Sphere,2-Cylinder,3-Three fingers,4-Two fingers,5-Sides,6-Cusp,7-Phone

Figure 5. The action sample of VAR amplitude

characteristics under different levels of grip force

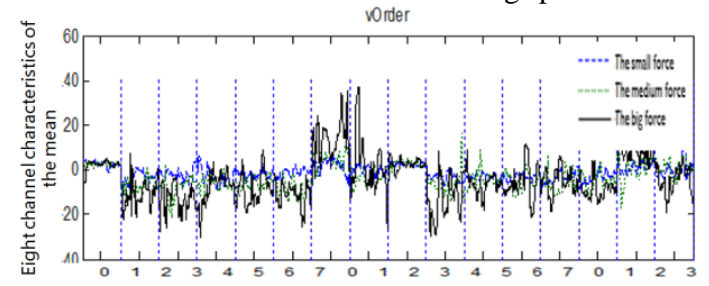

0-Free,1-Sphere,2-Cylinder,3-Three fingers,4-Two fingers,5-Sides,6-Cusp,7-Phone

Figure 7. The action sample of vOrder amplitude characteristics under different levels of grip force

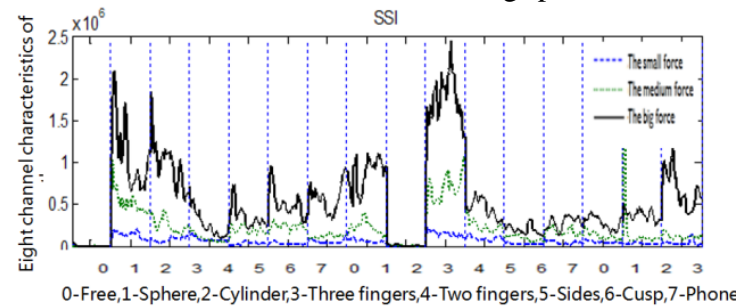

Figure 9. The action sample of SS1 amplitude characteristics under different levels of grip force

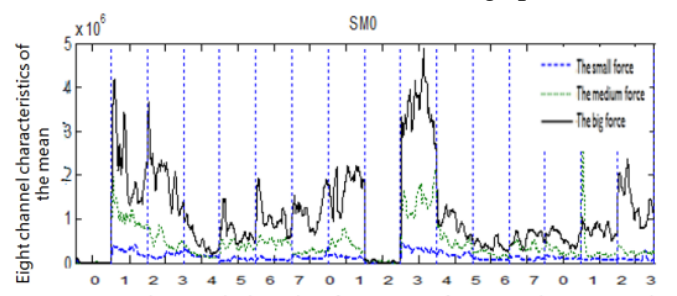

0-Free,1-Sphere,2-Cylinder,3-Three fingers,4-Two fingers,5-Sides,6-Cusp,7-Phone

Figure 11. The action sample of SM0 amplitude characteristics under different levels of grip force

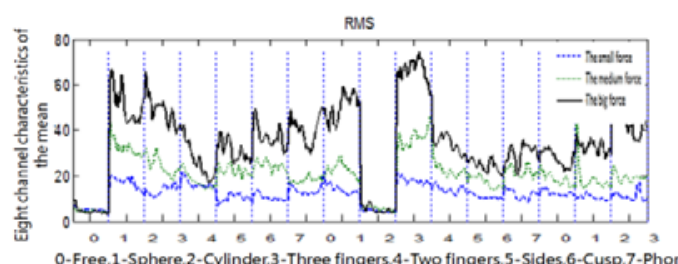

Figure 6. The action sample of RMS amplitude characteristics under different levels of grip force

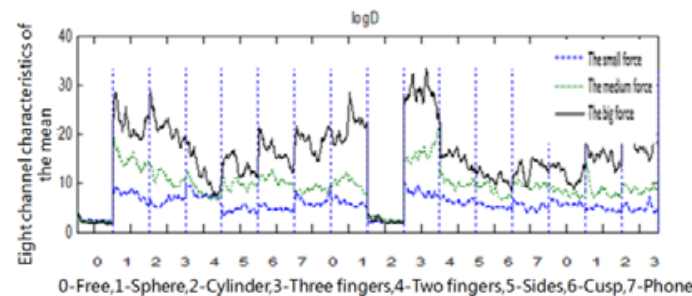

Figure 8. The action sample of $\log \mathrm{D}$ amplitude characteristics under different levels of grip force

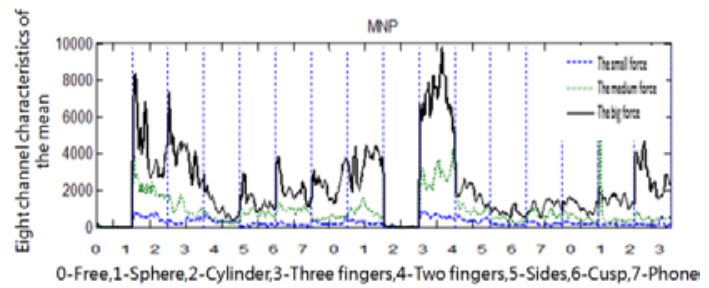

Figure 10. The action sample of MNP amplitude characteristics under different levels of grip force

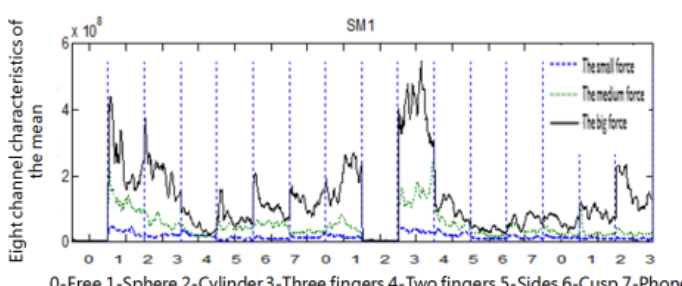

Figure 12. The action sample of SM1 amplitude characteristics under different levels of grip force

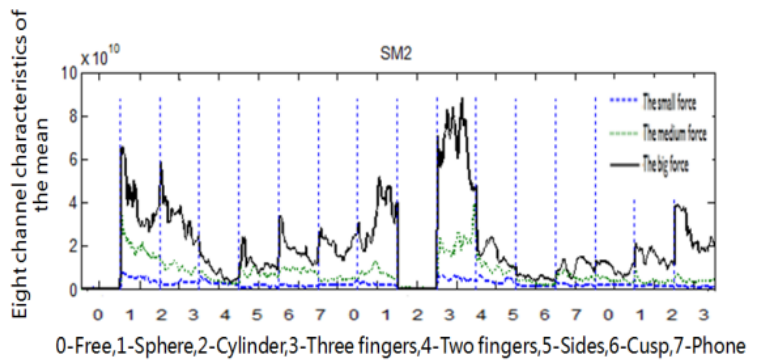

Figure 13. The action sample of SM2 amplitude characteristics under different levels of grip force 


\subsection{Searching optimal representative EMG features}

The above characteristic values are calculated based on the forearm EMG signal of the same subject. From Figure 7, the Order detector cannot distinguish the grasping force level (level of muscle contraction) correctly, other characteristic basically able to distinguish the level of muscle contraction of each action. The time-domain characteristic IEMG, MAV, MAV1, MAV2, VAR, RMS, logD and SSI are calculated based on the raw sEMG amplitude in analysis window. As shown from Figure 1 to Figure 6, Figure 8 and Figure 9 shows that their change trends are very closed; MNP, SM0, SM1 and SM2 characteristics are obtained by power spectrum after the EMG signal are fourier transformed in analysis window. It also provides information of the level of muscle contraction, the change trend is basically identical with others that are calculated based on the characteristics of sEMG amplitude. But the calculation of the frequency domain characteristic is more complicated.

In addition, for the same action and the same level of muscle contraction, the energy characteristic value is changing. The greater the level of muscle contraction is, the more violent the characteristic value changes. EMG signal is random and non-stationary. Noticed that in the second hooked grasping, the characteristics amplitude from the front acting force samples are too large, especially for the characteristics of the VAR, SSI, MNP and SM0. sEMG characteristic value of medium force greatly exceeds the sEMG characteristic value of big force and these characteristics are sensitive to the singularity of sEMG signal.

\section{Method for grasping force estimation and experimental result analysis}

Through the extraction and analysis of the EMG signal feature, it is clear that most visible characteristics can be used to distinguish the levels of grip strength and their distinction degrees are similar. Temporal characteristics were calculated based on the sEMG signal amplitude, which is simpler than the calculating frequency characteristic using the Fourier transform. Thus, in this paper three representative characteristics [11, 12], which are MAV, RMS and $\log D$, are Selected to decode the surface EMG by taking 8-channels sEMG characteristics at the same time.

The objective of decoding the discrete EMG signal is to find out the relationship between the movement of prosthesis and the grade of its force level. In order to achieve this goal, the same level of the grip strength (muscle contraction) is regarded as a category. Then, different levels of grip strengths can be sorted out by threshold judgment to relevant characteristics or pattern recognition methods.

Characteristic amplitude at different levels of grasping force was observed, where MAV features was selected as a representative. MAV of EMG feature for participants 5 is shown in Figure 15, suggesting that MAV characteristic value of different levels of the grasping force can be divided into three levels for the same grasping mode. As for the different grasping mode, MAV amplitude levels are different from the same level of the grasping force. That is to say, if taking the same group of threshold value into judging the grasping force level for the grasping process, the precision rate of force decoding will be far from satisfactory; while if setting a group of threshold value for every species mode, the precision rate of discrete force decoding will be significantly improved, but the training range for samples will increase greatly because the size of the decided threshold value is determined by training sets, in which every grasping mode is trained using three levels of grasping force.

As shown in Figure 14, a trainer performed two kind of online EMG control experiment with different levels of gripping force----cylindrical gripping and spherical gripping. The experiment includes 8 stages: 10s for natural stage; 10s for small force gripping; 10s for moderate force gripping; 10s for large force gripping; another 10s for natural stage; 10s for small force gripping; $10 \mathrm{~s}$ for moderate force gripping; and 10s for large force gripping. According to the training data set, the threshold of EMG signal decoding set to $\varepsilon_{1}=6, \varepsilon_{2}=12$ and $\varepsilon_{3}=22$, it is demonstrated the EMG force control of prosthetic hand would be carried out. However, there still shows poor stability of decoding of gripping force. 


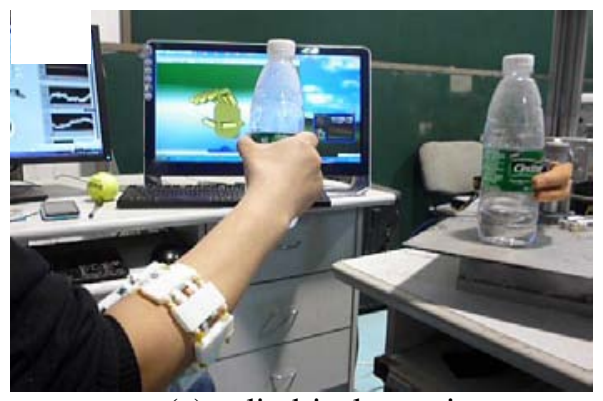

(a) cylindrical grasping

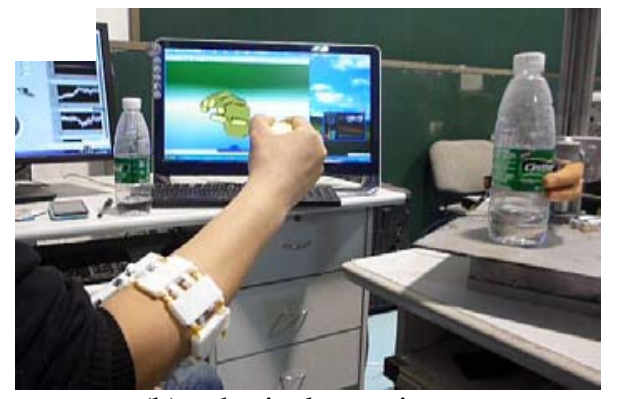

(b) spherical grasping

Figure 14. Experimental scene of EMG force control of prosthetic hand based on movement pattern and real-time decoding of grasping force

In this article the same group threshold of MAV () was used to distinguish the levels of grasping force for the movement pattern in Figure 15, and the results are shown in Figure 16, in which the range of 1-800, 801-1600 and 1601-2400 are corresponding to the small, medium and strong forces used in the grasping test respectively. In the results of experiments, natural state of MAV features value range is , and its accuracy is $96.94 \%$; as for small, medium and strong grasping force, their threshold values ranges and accuracy are , 73.06\%, 69.72\%, and , 71.39\% respectively. For the whole test set, 2400 actions were measured in which accuracy of determined grasping force level is $78.79 \%$, and if the action takes no account of natural states of the action modes into consideration, the accuracy of determined grasping force level will be $75.59 \%$.

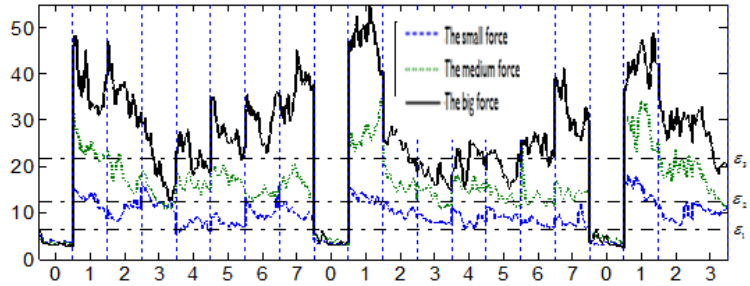

0-Free,1-Sphere,2-Cylinder,3-Threefingers,4-Twofingers,5-Sides,6-Cu sp,7-Phone

Figure 15. The MAV characteristics value of grasp experiment in different levels of the 5th tester

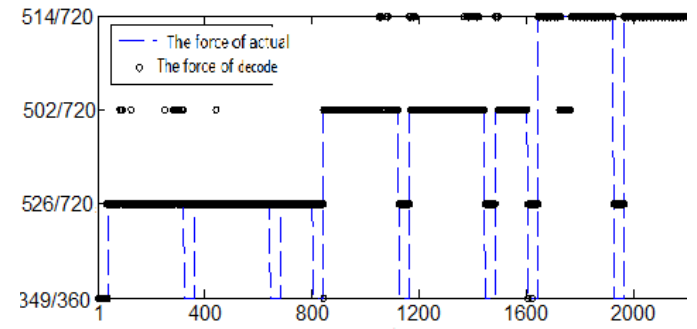

Figure 16. The results of the decoding threshold detection

Table 1.The correct rate of force decoding of ten subjects

\begin{tabular}{|c|c|c|}
\hline Subjects & MAV Threshold & Accuracy of force decoding \\
\hline Subject1 & $\varepsilon_{1}=7 \quad \varepsilon_{2}=12.5 \quad \varepsilon_{3}=20$ & $55.39 \%$ \\
\hline Subject2 & $\varepsilon_{1}=5 \quad \varepsilon_{2}=12$ & $74.61 \%$ \\
\hline Subject3 & $\varepsilon_{1}=5 \quad \varepsilon_{2}=11$ & $80.69 \%$ \\
\hline Subject4 & $\varepsilon_{1}=6 \quad \varepsilon_{2}=14$ & $71.13 \%$ \\
\hline Subject5 & $\varepsilon_{1}=6 \quad \varepsilon_{2}=12 \quad \varepsilon_{3}=22$ & $75.59 \%$ \\
\hline Subject6 & $\varepsilon_{1}=6 \quad \varepsilon_{2}=12.5 \varepsilon_{3}=22.5$ & $69.75 \%$ \\
\hline Subject7 & $\varepsilon_{1}=3 \quad \varepsilon_{2}=9$ & $81.37 \%$ \\
\hline Subject8 & $\varepsilon_{1}=2 \quad \varepsilon_{2}=5$ & $92.45 \%$ \\
\hline Subject9 & $\varepsilon_{1}=5 \quad \varepsilon_{2}=15 \quad \varepsilon_{3}=22.5$ & $69.75 \%$ \\
\hline Subject10 & $\varepsilon_{1}=2.5 \quad \varepsilon_{2}=6 \quad \varepsilon_{3}=12$ & $73.38 \%$ \\
\hline $\begin{array}{l}\text { Average accuracy } \\
\text { rate }\end{array}$ & \multicolumn{2}{|c|}{$74.41 \pm 9.61 \%$} \\
\hline
\end{tabular}

Similarly, discrete force decoding for other participants can also follow this method. It is noted that the set MAV threshold is different for each participant, which is shown in Table1; and the set 
threshold is estimated according to the known samples (train sets). In practice, according to the amputee's muscle, a reasonable threshold is determined to distinguish the different levels of muscle contraction.

From the results of Table 1, large individual differences can be found in the decoding method of discrete force in which threshold value was used to judging. While average accuracy for force decoding was found to be $74.41 \pm 9.61 \%$, and the testing sample in experiments is the entire population (altogether has 2040 action samples, not including natural state). Comparing to the force decoding method based on mode recognition, the accuracy of foregoing method is enough to meet the requirements. The greatest advantage of threshold judgment approach is that amputation patients do not need training on different muscle contraction level at each grasping mode, yet require estimating the value of threshold according to the result of different levels of muscle training at any one of movement mode. It is clear that decoding algorithm of the threshold judgment is much easier than the pattern recognition method, practically, the value of threshold can be adjusted according to the circumstances, which can be applied to the real-time control of the myoelectric prosthesis.

\section{Conclusion}

In this paper, the method of threshold value judgment is proposed to realize the discrete force estimation. The method is based on the level of sEMG's mean absolute value (MAV). The estimation of the grasping force can be achieve through the small training sample set (offline experimental analysis, the sample number accounted for $16.7 \%$ of the total sample number)and the short training time. It indicates that the strategy to estimat the grasping force from sEMG simultaneously is effective. Although the way of force estimation is not stable which caused by sEMG's non-stationary, and can be improved by the follow-up study.

\section{Acknowledgements}

This work was supported by National Basic Research Program of China (2011CB013302).

\section{References}

1. Zardoshti-Kermani M, Wheeler B C, Badie K, et al. EMG feature evaluation for movement control of upper extremity prostheses [J]. IEEE Transactions on Rehabilitation Engineering, 3(4): 324-333(1995)

2. Phinyomark A, Phukpattaranont P, Limsakul C. Feature reduction and selection for EMG signal classification [J]. Expert Systems with Applications, 39(8): 7420-7431(2012).

3. Lloyd D G, Besier T F. An EMG-driven musculoskeletal model to estimate muscle forces and knee joint moments in vivo [J]. Journal of biomechanics, 36(6): 765-776(2003).

4. Clancy E A, Hogan N. Estimation of joint torque from the surface EMG [C]. Proceedings of the Engineering in Medicine and Biology Society, 1991 Vol 13: 1991, Proceedings of the Annual International Conference of the IEEE: IEEE, 1991: 877-878.

5. Liu M M, Herzog W, Savelberg H H. Dynamic muscle force predictions from EMG: an artificial neural network approach [J]. Journal of electromyography and kinesiology, 9(6): 391-400(1999,).

6. Choi C, Kwon S, Park W, et al. Real-time pinch force estimation by surface electromyography using an artificial neural network [J]. Medical engineering \& physics, 32(5): 429-436(2010).

7. Castellini C, Gruppioni E, Davalli A, et al. Fine detection of grasp force and posture by amputees via surface electromyography [J]. Journal of Physiology-Paris,103(3): 255-262(2009).

8. Kuiken T A, Li G, Lock B A, et al. Targeted muscle reinnervation for real-time myoelectric control of multifunction artificial arms [J]. Jama, 301(6): 619-628(2009). 
9. Huang H-P, Chen C-Y. Development of a myoelectric discrimination system for a multi-degree prosthetic hand [C]. Proceedings of the Robotics and Automation, 1999 Proceedings 1999 IEEE International Conference on: IEEE, 1999: 2392-2397.

10. Oskoei M A, Hu H. Support vector machine-based classification scheme for myoelectric control applied to upper limb [J]. IEEE Transactions on Biomedical Engineering, 55(8): 1956-1965 (2008).

11. Kim K S, Choi H H, Moon C S, et al. Comparison of k-nearest neighbor, quadratic discriminant and linear discriminant analysis in classification of electromyogram signals based on the wrist-motion directions [J]. Current applied physics, 11(3): 740-745(2011).

12. Park S-H, Lee S-P. EMG pattern recognition based on artificial intelligence techniques [J]. IEEE Transactions on Rehabilitation Engineering, 6(4): 400-405(1998). 